\title{
PERMANENT MILLIARCSECOND LINK OF CELESTIAL AND TERRESTRIAL REFERENCE SYSTEMS
}

\author{
M. Feissel (Observatoire de Paris, Central Bureau of IERS)
}

\begin{abstract}
The celestial reference system and the terrestial reference system of the International Earth Rotation Service (IERS) are realized on the basis of observation programs in Very Long Baseline radio Interferometry and laser ranging to the Moon and artificial satellites. The celestial frame is materialized by the equatorial coordinates of radio sources observed in VLBI; the terrestrial frame is materialized by the cartesian coordinates of the terrestrial sites monitored by the three techniques. Series of the Earth Orientation Parameters are derived from the same observations. These series provide a permanent link between the celestial system and the terrestrial system at the level of $0.001 "$.

The global adjustment in which the reference systems are defined and realized is described, and the metrological properties of the frames and of the derived EOP are evaluated.
\end{abstract}

\section{Introduction}

In 1985, when IAU decided to create the new International Earth Rotation Service and endorsed the recommendations of the MERIT Working Group (Wilkins and Mueller, 1986), it was recognized that the accurate determination of the Earth's orientation makes it necessary that the appropriate terrestrial and celestial frames be monitored in a consistent fashion with the series of Earth Orientation Parameters (EOP).

The operation of IERS is based on observation and analysis by space geodesy techniques: VLBI, Lunar Laser Ranging (LLR), Satellite Laser Ranging (SLR); the colocation of some of the stations in common terrestrial sites allows the setting up of a terrestrial reference frame by combining into a common system the coordinates estimated from the various techniques of observation. The celestial reference frame is established on the extragalactic compact radio sources observed in the VLBI programs participating to IERS. The EOP are those angles which describe the orientation of the Celestial Ephemeris Pole in the terrestrial system and in the celestial system $(x, y ; d \psi, d \varepsilon)$, and the orientation angle of the Earth around this axis (UT1-TA1), as a function of time. The time series of the EOP thus provides a permanent tie between the IERS Terrestrial Reference Frame and the IERS Celestial Reference Frame. The accuracy of this link can be evaluated by considering vario'ss aspects: the stability of the two realized reference systems, the stability of the EOP evaluations, and their consistency with the reference frames.

\section{The observations and their analysis}

The observations are organized in networks, which have some sites in common. Table 1 summarizes the contribution of the various techniques to the three objectives of IERS. Although GPS observations are planned to be incorportated in IERS only after 1991, their expected performances are listed for reference. 
Table 1. Participation of the various techniques to IERS objectives. Numbers of fiducial points are given in the case of reference frames; in the EOP columns, the number of * reflects a compound of the precision, accuracy and density of measurements.

\begin{tabular}{|c|c|c|c|c|c|c|}
\hline $\begin{array}{l}\text { Observed } \\
\text { objects }\end{array}$ & Technique & $\begin{array}{l}\text { Terrestrial } \\
\text { frame } \\
\text { stations }\end{array}$ & $\begin{array}{l}\text { Celestial } \\
\text { frame } \\
\text { sources }\end{array}$ & $\begin{array}{l}\quad \text { Ear } \\
\text { celest. } \\
\text { pole }\end{array}$ & $\begin{array}{l}\text { Orientat } \\
\text { terrest. } \\
\text { pole }\end{array}$ & UT \\
\hline $\begin{array}{l}\text { Extragalactic } \\
\text { radio sources }\end{array}$ & VLBI & $3-40$ & $50-250$ & $* * *$ & $* * *$ & $* * *$ \\
\hline $\begin{array}{l}\text { Lunar } \\
\text { reflectors }\end{array}$ & LLR & $3-5$ & (Lun.eph) & $* *$ & & $* *$ \\
\hline GPS & $\begin{array}{l}\text { radio signals } \\
\text { from satellites }\end{array}$ & $20-200$ & (Sat.eph) & & $* *$ & $*$ \\
\hline $\begin{array}{l}\text { Satellite } \\
\text { reflectors }\end{array}$ & SLR & $40-100$ & (Sat.eph) & & $* * *$ & $* *$ \\
\hline
\end{tabular}

Analysis centres specialized in the various techniques analyse the observations and submit to the Central Bureau of IERS sets of results comprising a terrestrial frame (set of station coordinates) and the corresponding time series of the EOP over several years. In the case of VLBI, the celestial frames are also submitted, under the form of sets of estimated equatorial coordinates of extragalactic radio sources. A selection of these ensembles of results is combined in order to derive realizations of the IERS terrestrial and celestial systems and time series of EOP, following the concepts developped by Boucher et al. (1988).

The mutual consistency of the frames and EOP series was set originally at the end of the first year of operation of IERS, and the results were published in the Annual Report for 1988. The system was initialized under the form of the terrestrial and the celestial system. The series of EOP was attached to them by deriving, from the rotation angles of individual frames with respect to the IERS ones, biases for the corresponding time series of EOP (see Section 5); the series could then be combined into a homogeneous one. This global analysis is made possible by the high degree of consistency in the astronomical and geophysical modelling used by the various analysis centres which participate in it, in particular through the reference to the IERS Standards (McCarthy, 1989).

\section{The IERS Terrestrial Reference System}

The principles on which the IERS Terrestrial Reference System is established and maintained are described by Boucher and Altamimi (1990a).

It is geocentric, the centre of mass being defined for the whole Earth, including oceans and atmosphere. Only observations which can be modelled by dynamical techniques (presently SLR and LLR for IERS) can determine the center of mass. The VLBI results can be referred to a geocentric system by adopting for a station its geocentric position at a reference epoch as provided from external information.

The unit of length is the metre (SI). The scale is that of a local Earth frame, in the sense of a relativistic theory of gravitation. It is obtained by appropriate relativistic modelling, particularly for VLBI and LLR which are usually modelled in a barycentric frame. 
The directions of axes are consistent with those of the previous conventional terrestrial reference frame (CIO/BIH pole and $\mathrm{BIH}$ origin of longitudes) within the uncertainty of the latter.

As new site coordinates become available, as new observing techniques provide terrestrial frames, or as improved modelling is developped, new realizations of the frame are constructed. To insure the constancy of the direction of axes relative to the Earth's crust, appropriate treatment for the internal consistency of the site positions and motions is applied. Then the axes of the new frame are rotated to those of the preceding realization. Through this procedure, the frame is constantly improved and extended, while all successive realizations give access to the same conventional system. Checks on the successive versions indicate that the constancy of axes directions is insured within \pm $0.0005^{\prime \prime}$.

The terrestrial reference system is made available by the site coordinates (119 in the 1990 realization, see Boucher and Altamimi, 1990b), or by the transformation parameters of individual terrestrial frames relative to the IERS ones. As an example, the IERS Annual Report for 1989 gives this information for 18 different terrestrial frames. The system is used as a common reference in worldwide applications, such as time transfer by satellite links or the connection of tide gauges for the study of mean sea level.

\section{The IERS Celestial Reference System}

The definition of the IERS celestial reference system and the maintenance process of the associated frame are similar to those of the terrestrial system and frame.

The IERS Celestial Reference Frame (ICRF) is established at the Central Bureau of IERS on the basis of extragalactic reference frames obtained by the IERS Analysis Centres for VLBI (in 1990: GSFC, JPL, NGS, USNO). These centres use consistent standards in order to make their frames barycentric, and they impose the condition that the sources do not move relative to one another. Appropriate procedures are applied so that the source coordinates are unaffected by the inaccuracy of the precession-nutation model, except for arbitrary offsets in the reference poles. The use of the standard precession-nutation model in computing the coordinates at J2000.0 introduces a small offset of the pole of the frame relative to the real position of the mean pole. The various ways in which the right ascension origin is fixed also introduce small offsets. The above mentionned offsets are modeled in the Central Bureau combination as three rotation angles for each individual frame relative to the IERS one; they are in general smaller than three milliarcseconds.

As new information on source positions becomes available, new realizations of the ICRF are introduced, insuring that a new realization has globally no rotation with respect to the previous one. The maintenance process includes the selection of primary sources on which the no rotation condition is applied; this procedure aims at improving the source coordinates and at extending the list of sources (primary and others), while keeping the initial direction of the axes. The 1990 version (IERS Annual Report for 1989) includes a total of 228 radio sources, among which 51 are primary; 113 sources have position uncertainties under $0.001^{\prime \prime}$ (vs. 76 in the previous realization) and the rotation angles relative to the previous realization are insignificant at the level of 0.0001 ".

Details on the IERS celestial reference system and frame are given by Arias and Feissel (1990a, 1990b). A tentative evaluation of the consistency of the IERS celestial system with the FK5 is given in the Annex. We conclude that the directions of axes of the IERS celestial system are in agreement with those of the FK5 within the uncertainty of the latter. 


\section{The Earth Orientation parameters}

Comparing pairs of terrestrial frames or pairs of celestial frames, one can predict the biases between the respective EOP series $(\Delta x, \Delta y, \Delta U T 1, \Delta d \psi, \Delta d \varepsilon)$. Let $R_{1}, R_{2}$, and $R_{3}$ be the rotation angles between two terrestrial frames, reckoned respectively around the Ox axis (in the direction of the prime meridian), the Oy axis (longitude $90^{\circ} \mathrm{E}$ ) and the $\mathrm{Oz}$ axis (polar axis); let $\mathrm{A}_{1}, \mathrm{~A}_{2}$, and $\mathrm{A}_{3}$ be the rotation angles between two celestial frames (see definition in Annex.) The predicted biases in EOP's are as follows (Zhu and Mueller, 1983).

$$
\begin{aligned}
& \Delta x=R_{2}, \quad \Delta y=R_{1}, \\
& \Delta \mathrm{UT} 1=A_{3}-R_{3}, \\
& \Delta d \psi=A_{2} / \sin \varepsilon, \Delta d \varepsilon=-A_{1} .
\end{aligned}
$$

Table 2 gives the average discrepancies between the predicted and observed differences between time series, for VLBI and SLR. The discrepancies are in the range $0.0002 "$ $0.002 "$.

Table 2. Average discrepancies between reference frames relative rotations and relative biases in EOP series. The uncertainties listed refer to the individual estimates of the discrepancies. Unit: $0.001 "$.

\begin{tabular}{l|ccccc}
\hline \multirow{2}{*}{ Techniques } & \multicolumn{5}{|c}{ Earth Orientation Parameters } \\
& $\mathrm{x}$ & $\mathrm{y}$ & $\mathrm{UT} 1$ & $\mathrm{~d} \psi \sin \varepsilon$ & $\mathrm{d} \varepsilon$ \\
\hline VLBI-VLBI & $0.7 \pm 0.6$ & $1.5 \pm 0.6$ & $0.3 \pm 0.6$ & $0.4 \pm 0.2$ & $0.2 \pm 0.2$ \\
SLR-SLR & $0.2 \pm 0.6$ & $0.7 \pm 0.6$ & & & \\
VLBI-SLR & $1.8 \pm 1.1$ & $1.5 \pm 1.0$ & & & \\
\hline
\end{tabular}

The comparisons of Table 2 are derived at a common epoch (1988.0). Provided that two series of the pole coordinates and UT1 are referred to the same site motion model, the difference of two series of EOP should not change with time. A check of the relative linear drifts indicates that the unexplained relative drifts in any of the five EOP are generally insignificant (absolute value lower than 0.0001 "/year), with some exceptions up to the level of $0.0005^{\prime \prime} /$ year.

The two above evaluations set an upper limit at the level of $0.001^{\prime \prime}$ to the accuracy of the best Earth orientation results. The precision of the time series, i.e., the stability of their measurement errors, can be estimated from comparisons two by two with the help of the Allan variance. In an earlier study (Feissel, 1990), we showed that the individual series have a white noise error spectrum for sampling times of five days $\left(0.001^{\prime \prime}\right)$ through one year $\left(0.0001^{\prime \prime}\right)$. The IERS series of EOP, which is obtained by combining several of these series, is expected to have also a white noise error spectrum, at a somewhat better level. 


\section{Summary}

The IERS terrestrial and celestial systems are realized by sets of fiducial points which coordinates have a precision better than 0.001" and an accuracy of 0.001-2"; their axes are maintained within $\pm 0.0005 "$.

The time series of the EOP which relate them have a measurement stability of 0.001 " (one day interval) to 0.0001 " (one year); their link to the reference system is known within $0.001 "$.

Figure 1 shows these uncertainties, together with an estimation of the natural unstability of the Earth's rotation. UT1-TA1 has a stability of about \pm 0.006 " at one day interval, with a very sharp increase for longer time intervals; the polar motion spectrum starts at $0.0015^{\prime \prime}$ for a five day interval, with a relatively rapid rise for longer time intervals; the celestial pole coordinates have a flicker noise spectrum, at the level of $0.0006 "$ ".

In conclusion, the observations collected and treated in the operation of IERS give a permanent tie at the level of 0.001 " between its terrestrial system and its celestial system. The realization of the reference systems as well as their ties are unaffected by the geophysical noise in the Earth's rotation.

Figure 1. Spectra of the Earth's rotation irregularities and of their measurement.

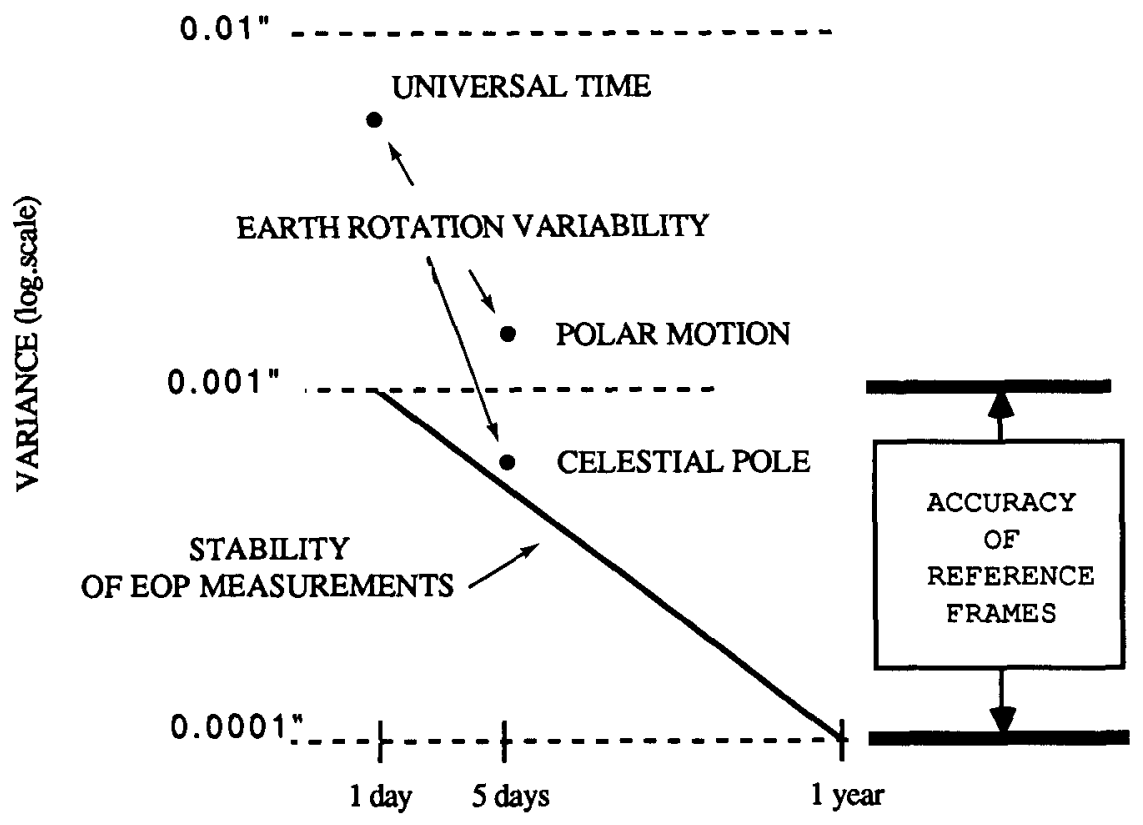

SAMPLING TIME (log.scale) 


\section{Annex. Relationship between the FKS and IERS axes.}

The figure represents estimated positions of the FK5 and IERS polar axes relative to the mean celestial pole at J2000.0, and estimated relative positioning of the FK5 and IERS right ascension origins. The estimated parameters are rotation angles $A_{1}, A_{2}$, and $A_{3}$ around three axes in the directions $\alpha=0^{\mathrm{h}}, \delta=0 ; \alpha=6^{\mathrm{h}}, \delta=0 ; \delta=90^{\circ} . A_{1}, A_{2}$, are plotted in a plane tangent to the celestial sphere at the celestial pole; $A_{3}$ is plotted in a plane tangent to the celestial sphere in the vicinity of the equinox.
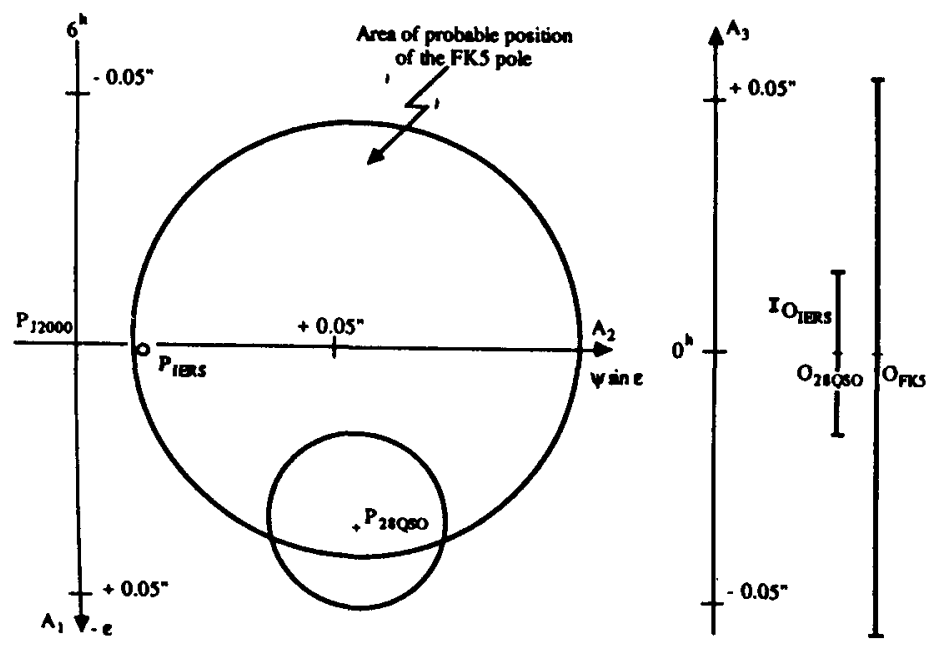

Pole. The coordinate axes are centered at the mean celestial pole at J2000.0. $\mathrm{P}_{\mathrm{IERS}}$ is an estimation of the IERS polar axis derived from VLBI analyses (e.g. Steppe et al., 1990); the various VLBI estimations agree within 0.001" (radius of the circle around $\left.\mathrm{P}_{\text {IERS }}\right)$. The area of probable position of the FK5 pole is obtained by first considering that the systematic part is dominated by a correction of -0.25 "/cy to the precession constant imbedded in the FK5 System, and second by adopting Fricke's (1982) estimation of the accuracy of the FK5 equator ( \pm 0.02"), and Schwan's (1988) estimation of the limit of the residual rotation ( $\pm 0.07 " / c y)$, taking the epochs of observations from Fricke et al., (1988). If one assumes that the error in the precession rate is absorbed by the proper motions of stars, the circle has to be centered at $\mathrm{P}_{\mathrm{J} 2000}$, keeping the same radius. $\mathrm{P}_{280 S O}$ and its error circle are derived from a comparison of the coordinates of 28 quasars in the FK5 System (Ma et al., 1990) with their coordinates in the IERS Celestial Reference System.

Origin of right ascensions. $\mathrm{O}_{280 \mathrm{so}}$, corresponding to the origin of the right ascensions of 28 quasars in the FK5 System given by Ma et al. (1990), is set arbitrarily as the origin. The position of $\mathrm{O}_{\text {IERS }}$ is derived from a least squares adjustment between the 28 quasars FK5 right ascensions and the IERS ones; the error bar on $\mathrm{O}_{280 \text { so }}$ is derived from this adjustment. The error bar on $\mathrm{O}_{\text {IERS }}$ results from an estimation of the stability of the IERS origin of right ascensions.

$\mathrm{O}_{\mathrm{FK} 5}$ is set at the same position as $\mathrm{O}_{28 \mathrm{Q} S}$; the error bar is derived from the quadratic sum of the accuracies given by Fricke $\left( \pm 0.045^{\prime \prime}\right)$ and Schwan $( \pm 0.07 " / \mathrm{cy})$, considering a mean epoch of 1955 for the proper motions in right ascension. 


\section{References}

Arias, E.F., and Feissel, M., 1990a: The celestial system of the International Earth Rotation Service. Proc. IAU Coll. 141, 119, Kluwer.

Arias, E.F., and Feissel, M., 1990: The celestial system and frame of IERS. This volume.

Boucher, C. and Altamimi, Z., 1990a: A conventional terrestrial system consistent with WGRS. This volume.

Boucher, C., and Altamimi, Z., 1990b: ITRF89 and other realizations of the IERS Terrestrial Reference System for 1989. IERS Technical Note No. 6. Paris Observatory.

Boucher, C., Feissel, M., and Lestrade, J.-F., 1988: Concepts and methods of the Central Bureau of the International Earth Rotation Service. Bull. Géod., 62, 511.

Feissel, M., 1990: Accuracy of the determination or prediction of Earth Orientation Parameters. Proc. IAG Symp. 105. Springer-Verlag.

Fricke, W., 1982: Determination of the Equinox and Equator of the FK5, Astron. Astrophys., 107, L13-16.

Fricke, W., Schwan, H., and Lederle, T., 1988. Fifth Fundamental Catalogue, Part I. Veröff. Astron. Rechen Inst. Heidelberg, 32.

IERS, 1990: Annual Report for 1989. Paris Observatory.

Ma, C., Shaffer, D.B., De Vegt, C., Johnston, K.J., and Russell, J.L., 1990: A radio optical reference frame I., Astron. J., 99, 1284-1298.

McCarthy, D.D., (ed.), 1989: IERS Standards (1989). IERS Technical Note No. 3, Paris Observatory.

Schwan, H., 1988: Precession and galactic rotation in the system of the FK5, Astron. Astrophys., 198, 116-124.

Steppe, J.A., Oliveau, S.H., and Sovers, O.J., 1990: Earth rotation parameters from DSN VLBI, IERS Technical Note No. 5, Paris Observatory.

Wilkins, G.A., and Mueller, I.I., 1986: On the rotation of the Earth and the terrestrial reference system: Joint summary report of the IAU/IUGG Working Groups MERIT and COTES. Bull Géod., 60, 85.

Zhu, S.Y., and Mueller, I.I., 1983: Effects of adopting new precession, nutation and equinox corrections on the terrestrial reference frames. Bull. Géod., 54, 29. 